University of Nebraska - Lincoln

DigitalCommons@University of Nebraska - Lincoln

5-8-2006

\title{
Magnetic Force Microscopy Study of CoPtCrO Perpendicular Media With Superparamagnetic And Permanent Magnet Tips
}

\author{
L. Yuan \\ University of Nebraska - Lincoln \\ Lei Gao \\ University of Kansas, Igao3@unl.edu \\ L. Nicholl \\ University of Nebraska - Lincoln \\ Sy-Hwang Liou \\ University of Nebraska-Lincoln, sliou@unl.edu
}

M. Zheng

MMC Technology, San Jose, CA, USA

See next page for additional authors

Follow this and additional works at: https://digitalcommons.unl.edu/physicsliou

Part of the Physics Commons

Yuan, L.; Gao, Lei; Nicholl, L.; Liou, Sy-Hwang; Zheng, M.; Abarra, E. N.; Acharya, B. R.; Choe, G.; Malhotra, S. S.; and Han, B., "Magnetic Force Microscopy Study of CoPtCrO Perpendicular Media With Superparamagnetic And Permanent Magnet Tips" (2006). Si-Hwang Liou Publications. 96. https://digitalcommons.unl.edu/physicsliou/96

This Article is brought to you for free and open access by the Research Papers in Physics and Astronomy at DigitalCommons@University of Nebraska - Lincoln. It has been accepted for inclusion in Si-Hwang Liou Publications by an authorized administrator of DigitalCommons@University of Nebraska - Lincoln. 


\section{Authors}

L. Yuan, Lei Gao, L. Nicholl, Sy-Hwang Liou, M. Zheng, E. N. Abarra, B. R. Acharya, G. Choe, S. S. Malhotra, and B. Han 


\section{Magnetic Force Microscopy Study of CoPtCrO Perpendicular Media With Superparamagnetic And}

Permanent Magnet Tips

Yuan, L.

Gao, L.

Nicholl, L.

Liou, S. H.

Zheng, $\mathrm{M}$.

Abarra, E. N.

Acharya, B. R.

Choe, G.

Malhotra, S. S.

Han, B.

Department of Physics \& Astronomy, University of Nebraska-Lincoln, Lincoln, NE, USA

This paper appears in:

Magnetics Conference, 2006. INTERMAG 2006. IEEE International

Publication Date: 8-12 May 2006

Pages: 893 - 893

Location: San Diego, CA, USA

ISBN: 1-4244-1479-2

Digital Object Identifier: 10.1109/INTMAG.2006.374924

Posted online: 2007-06-25 10:09:23.0

Copyright (C) 2006 IEEE. Used by permission. 


\section{HD-05}

Magnetic Force Microscopy Study of CoPtCrO Perpendicular Media With Superparamagnetic And Permanent Magnet Tips.

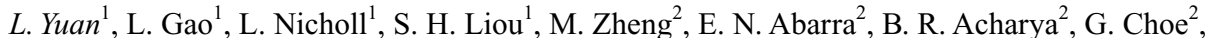
S. S. Malhotra ${ }^{3}$ and B. Han ${ }^{4}$

1. Physics \& Astronomy, University of Nebraska-Lincoln, Lincoln, NE, USA; 2. MMC

Technology, San Jose, CA, USA; 3. Komag, San Jose, CA, USA; 4. State Key Laboratory of

Magnetism, Institute of Physics, Chinese Academy of Science, Beijing, Beijing, China

Magnetic force microscopy (MFM) has been widely used in the study of magnetic recording media It requires high resolution MFM tips as well as better understanding of the obtained magnetic images. In this study, we compared the images obtained by superparamagnetic and permanent MFM tips, that allows us to explain the issues related to the frequency double in some of domain images of the recording media. We also investigated the domain structures of high density recording bits (up to $1100 \mathrm{kfci}$ ) written on ac and dc-erased $\mathrm{CoPtCrO}$ perpendicular magnetic recording (PMR) media with permanent magnet MFM tips under ambient conditions.

As shown in Fig. 1, we compared the magnetic (phase) image of the $200 \mathrm{kfci}$ track in an ac-erased area taking with a permanent and a superparamagnetic tip. Fig. 1a is the image obtained by the permanent magnet MFM tip, that shows a dominate spectral frequency of about $200 \mathrm{kfci}$. Fig. $1 \mathrm{~b}$ is the image taken by a superparamagnetic tip, that shows a dominate spectral frequency of about 400 kfci. This clearly demostrated that the frequency doubling were observed by using a superparamagnetic tip. This may explain the observation of the frequency doubling by Zhifeng Deng et. a [1] in their phase images of a PMR medium using metal-coated carbon nanotube tips. We show that the magnetic images obtained by different types of MFM tips can provide valuable magnetic information about the sample.

PMR has been demonstrated as a promising technology to sustain continuous growth in data storage capacity compared to longitudinal magnetic recording media. Two preconditioning techniques, ac and dc-erasure, can be performed before recording tracks on the medium. In this study, we investigated the domain structures of high density recording bits written on ac and dc-erased $\mathrm{CoPtCrO}$ PMR media with our permanent magnet MFM tips.

Fig. 2a shows the domain images of recording tracks with linear recording densities of 900 and $1100 \mathrm{kfci}$ written after ac-erasure. Tracks of 900 and $1100 \mathrm{kfci}$ correspond to bit sizes of 28 and 23 $\mathrm{nm}$, respectively. The MFM images of the $900 \mathrm{kfci}$ tracks present well-resolved recording bits. The $1100 \mathrm{kfci}$ transitions are more difficult to discern due to the strong background. Fig. $2 \mathrm{~b}$ shows the domain images of recording tracks with linear recording densities of 900 and $1100 \mathrm{kfci}$ written after dc-erasure. The bits corresponding to $900 \mathrm{kfci}$ are clearly visible. Some $1100 \mathrm{kfci}$ bits are observable but are modulated by the magnetic clusters. In order to get a more quantitative analysis of the tracks, we have performed a Fourier transform to get the power spectrum of the digitized profile. Fig. 3a shows the power spectra of the 900 and $1100 \mathrm{kfci}$ tracks written after ac-erasure. The peaks correspond to the recorded signal. Fig. $3 \mathrm{~b}$ shows the averaged power spectra for tracks recorded after dc-erasure. Comparing the Fourier spectra, the peak for $1100 \mathrm{kfci}$ written after acerasure has a larger amplitude than written after dc-erasure. Since the peaks are from the periodic

properties of the recording bits, this indicates the periodicity of the transition bits for $1100 \mathrm{kfci}$ after ac-erasure are better than after dc-erasure in this medium.

In summary, we show that the magnetic transition of $1100 \mathrm{kfci}$ density can be identified with our permanent magnet tips. These permanent magnet tips can be used to resolve magnetic features smaller than $25 \mathrm{~nm}$ in ambient conditions. To study of magnetic images, using more than one type of tips may help in the quantitative analysis of MFM data. [1] Zhifeng Deng, Erhan Yenilmez, Josh Leu, J. E. Hoffman, Eric W. J. Straver, Hongjie Dai, and Kathryn A. Moler, Appl. Phys. Lett., 85, 6263 (2004).

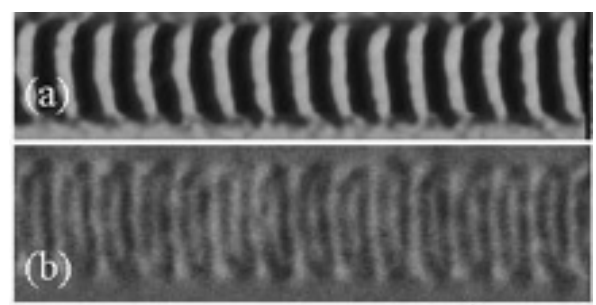

$1 \mu \mathrm{m}$

Fig. 1 Magnetic (phase) images of the 200 kfci track in an ac-erased area taking with (a) a permanent magnetic tip and (b) a superparamagnetic tip.
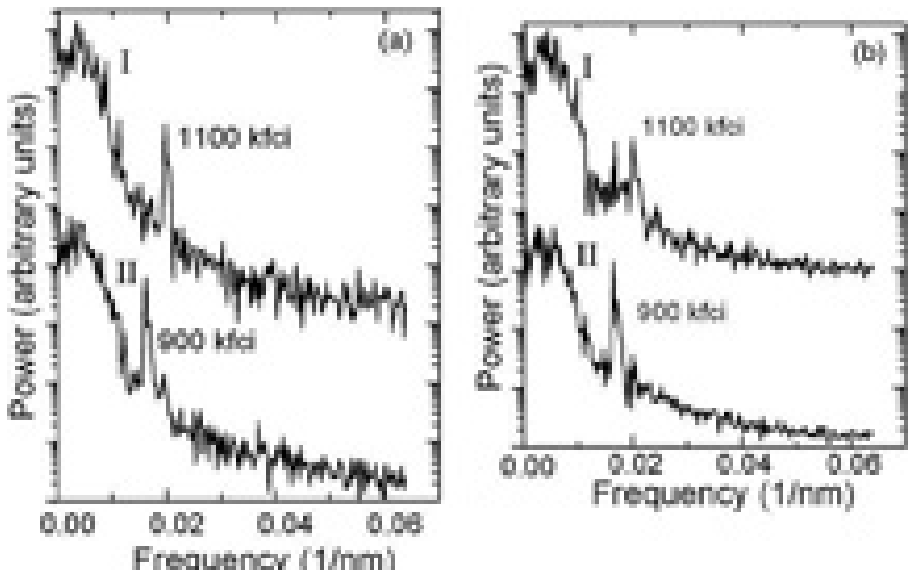

Fig. 3 Fast Fourier transform power spectra of the along-track profile for tracks written after (a) ac-erasure and (b) dc-erasure for the track densities of (I) $1100 \mathrm{kfci}$ and (II) $900 \mathrm{kfci}$. 